\title{
Challenging medical knowledge translation: convergence and divergence of translation across epistemic and cultural boundaries
}

John Ødemark ${ }^{1 凶} \&$ Eivind Engebretsen ${ }^{1}$

In this article, and the topical collection accompanying it, we aim to challenge socalled knowledge translation (hereinafter KT) in medicine and healthcare. The abbreviation ' $K T$ ' refers to a variety of scientific practices and research activities, bound together by the common goal of 'bridging the gap' between science in laboratories and clinical application, and, more generally, putting research-based knowledge into policy and practical care. Our objective, then, is to challenge KT by working through and with the convergence and divergences between different translational epistemologies. As KT has had a massive impact on practical healthcare, global health, and knowledge policy, as well as governance relating to sustainability, a critical examination of KT is of huge academic and societal significance. The point of departure for the contributors to this collection is the observation that $\mathrm{KT}$ is based upon a reductive understanding of translation and knowledge transmission. Standard models of KT take translation and knowledge transmission as a phenomenon for granted, and accordingly downplay the complexity of translation as an entangled material, textual and cultural process, which inevitably affects the 'original scientific message'. By contrasting KT with historical, cultural, and epistemic differences from its scientific 'prehistory', and by analysing it with reference to broader humanistic and material views of translation, we aim to develop concepts of medical translation that can cope with contemporary epistemic and cultural differences. 


\section{Introduction}

his collection aims to challenge so-called knowledge translation (hereinafter KT) in medicine and healthcare. The abbreviation ' $\mathrm{KT}$ ' refers to a variety of scientific practices and research activities bound together by the common goal of 'bridging the gap' between science in laboratories and clinical application, and, more generally, putting research-based knowledge into policy and practical care (Strauss et al., 2009; Woolf, 2008). KT has been particularly important in medicine but has also played an important role in other forms of science-based policy, for instance in climate change governance (Machen, 2018).

So-called translational research first emerged in the biomedical field in the 1990s. This research was, from the very beginning, explicitly presented as a solution to the challenge of slow and insufficient uptake of research discoveries in everyday clinical practice. Translational research thus emerged as a solution to what we can call a temporal and a quantitative problem; the flow from science to practice was too slow, and the amount of new knowledge transported from science to society too little. Accordingly, translational research set out to solve problems related to the social efficiency of biomedical research, the time lag between science and everyday practice in the clinic, and the quantitative dilemma concerning the amount of new medical knowledge turned into practice in the healthcare system (e.g., Mankoff et al., 2004).

$\mathrm{KT}$ is little concerned with the entanglement of the cultural and the biomedical aspects of knowledge. Hence, the view of translation in KT contrasts sharply with the celebration of difference now dominant in the human sciences. The turn to translation in the humanities attempts to tackle cultural and epistemic difference in a global age. Besides, the term translation has been applied to extra-lingual transfer, such as cultural translation-also encompassing social phenomena such as migration (Buden and Nowotny, 2009). These expansions of 'translation' beyond the linguistic can in fact be construed as a return to older, more material notions of translation. Pre-modern conceptualizations of translation, for instance, encompassed boundary crossings such as the translatio of Saints (referring both to the ritual transfer of holy bodies and the texts documenting them), and the translatio studii et imperii (the transference of power/knowledge from old to new empires) (Evans, 1998; Wintroub, 2015). As the concept of 'translation' and the crossing of epistemic, cultural, and linguistic boundaries have become increasingly important in the human sciences, one could think that KT formed a part of this new translational paradigm. The turn to translation in medicine is, however, of a radically different kind. It is aimed at preserving the identity of the scientific message, not at celebrating epistemic or cultural difference.

The objective of this collection, then, is to challenge KT by working through and with the convergence and divergences between different translational epistemologies. As KT has had a massive impact on practical healthcare, global health, and knowledge policy, as well as governance relating to sustainability, a critical examination of $\mathrm{KT}$ is of huge academic and societal significance. The point of departure for the contributors to this collection is the observation that $\mathrm{KT}$ is based upon a reductive understanding of translation and knowledge transmission. Standard models of KT take translation and knowledge transmission as a phenomenon for granted, and accordingly downplay the complexity of translation as an entangled material, textual and cultural process, which inevitably affects the 'original scientific message'. By contrasting KT with historical, cultural, and epistemic differences from its scientific 'prehistory', and by analyzing it with reference to broader humanistic and material views of translation, this collection aims to develop concepts of medical translation that can cope with contemporary epistemic and cultural differences.

In the rest of this introductory article, we will discuss how views on translation from translation studies, and the human sciences more broadly, can supplement current models of KT. Our guiding assumption is that such a supplement can lay the foundation for an expanded model of KT that is able to cope with epistemic and cultural differences, and the inevitable entanglement of the socio-cultural and the biomedical (cf. Engebretsen et al., 2017, 2020; Ødemark et al., 2021 ${ }^{1}$ ). Firstly, we will give a short description of $\mathrm{KT}$ as a translational practice, then we will sketch ways of supplementing KT with models of translation from other disciplines.

\section{$\mathrm{KT}$ as a kind of translation}

$\mathrm{KT}$, then, was devised as an answer to a problem of governance and efficacy: the need to implement new, scientifically warranted knowledge in medical and social practice. The assumption behind this was - and still is - that relevant, biomedical knowledge exists, but that there is a knowledge cleft between state-of-the-art scientific knowledge and the social places and institutions, like hospitals and clinics. Hence, the all-important task for KT, as a combined scientific and social instrument, is to reduce the gap between theory and practice by making medical practice knowledge based. We see this clearly in WHOs definition of KT:

Knowledge translation (KT) has emerged as a paradigm to address many of the challenges and start closing the "knowdo" gap. KT is defined as "The synthesis, exchange, and application of knowledge by relevant stakeholders to accelerate the benefits of global and local innovation in strengthening health systems and improving people's health." (World Health Organization, 2019. Ageing. Knowledge translation, our emphasis).

Here KT is construed as a 'paradigm' that addresses medical challenges. These challenges have everything to do with the opposition between theory and practice. The space that translation crosses is between science and social practice, and the objective of KT is to close the 'know-do gap'.

We can use this spatial definition of translation to relate $\mathrm{KT}$ to other forms of translation. Etymologically, translation is all about crossing spaces. ${ }^{2}$ While inter-lingual translation crosses the space between languages, KT aims to cross the space between biomedical knowledge and practical healthcare. Ideally, there should be an equivalence of some sort between the message produced by science and its application in practice, and in socio-cultural spaces like hospitals and clinics. In other words, the aim of KT as a form of translation is to close or bridge the gap between knowing and doing, and thus reduce the distance between these poles.

In anthropology, cultural studies and cultural history, the space crossed by translation is often defined as cultural, that is, as a space between different cultures. P. Burke has, for instance, observed that the concept of 'cultural translation' emerged 'to describe what happened in cultural encounters when each side tries to make sense of the action of the other' (Burke, 2007, p. 8, our emphasis). Indeed, questions concerning the commensurability of knowledge from different disciplines, places and times have also long been associated with 'translation' in disciplines the history and philosophy of science as well (often through an import from anthropology) (Severi and Hanks, 2015). In KT, however, there is little sustained reflection about the crossing of cultural and epistemological spaces. Nor has KT been accompanied by theoretical reflection on translation as a creative and performative process of knowledge production. 
While 'translation' has emerged as a key theoretical concept to deal with epistemic and cultural difference in the human sciences, KT denotes a scientific and purportedly non-cultural practice that defines the social and cultural as a 'barrier' to the transmission of knowledge already formulated in the laboratory and confirmed by randomized controlled trials. The materialization of 'translation' in medicine thus contrasts with the attention to epistemic and cultural difference and the productivity of texts in the humanities.

Bearing this lack of epistemological and socio-cultural reflexivity in mind, prominent medical researchers have questioned the epistemological aspects of KT and argued that the time has come to 'drop the knowledge translation metaphor' (Greenhalgh and Wieringa, 2011). While wholly in line with the epistemological diagnosis leading to this criticism, we will nevertheless claim that an expanded notion of the 'translation metaphor' might help us devise forms of KT more attuned to biological, epistemological, and cultural complexities. Thus, while we argue that KT is based on a simplistic view of translation and knowledge production, we also maintain that KT as a practical form of knowledge production and knowledge transmission might benefit from incorporating more theoretical notions of translation as an entangled material, textual and cultural process.

Current notions of $\mathrm{KT}$ as a translational practice actually resembles inter-lingual translation practices that aim for semantic and pragmatic equivalence between an original source text (ST) and the new target text (TT) (e.g., Nida and Taber, 1969). Using another analogy from inter-lingual translation, we could even say that the current construal of knowledge production and translation in KT comes close to the now deeply compromised literary view of translation where the translator's work is 'invisible' (Venuti, 1995), and/or the translator merely an 'ancillary' instance (Bergman, 1984) in the re-production of meaning and knowledge. Indeed, given this view of $\mathrm{KT}$, the translator is doomed to be a traitor-not of artistic genius but of the scientific logos - the 'evidence' found in the laboratory and confirmed by randomized controlled trials. In translation studies, in contrast, it is commonplace that the original source text (ST) can never be fully recovered by the target text (TT), that inter-lingual translation implies semantic shifts, and that the ST must be 'rewritten in domestic dialects and discourses, registers and styles' pertaining to the TT and target culture (TC) (Venuti, 2009).

KT rejects the productivity of translation. The translational paradigm established in so-called actor network theory (ANT) and science and technology studies (STS) offers yet another contrast to KT in terms of the productivity of translation. ANT and STS studies have asserted that the productivity of translation is the very condition for all knowledge and scientific effects. New work in the history of science has also been concerned with translation. By following 'science in action', empirically orientated scholars have come to focus their attention on the construction of science. STS and ANT have emphasized that translation is never simply a discursive process, but a material and cultural practice, conducted in complex contexts inhabited by variously interacting interests (Wintroub, 2015). B. Latour even maintains that his understanding of translation solves-or rather dissolves-the 'old' formulation of the issue of cross-disciplinary and cross-cultural commensuration. He presents a pragmatic solution that simply points to the fact that cultures and natures have always been translated, and that the activity of relating and translating is undertaken with reference to yardsticks that do not belong to the 'nature' of the things related, but to the instruments of commensuration. Instruments of translation are inevitably produced, and in practice, the problems of commensurability will be solved:

Worlds appear commensurable or incommensurable only to those who cling to measured measures. Yet all measures, in hard and soft science alike, are also measuring measures, and they construct a commensurability that did not exist before their own calibration. Nothing is, by itself, either reducible or irreducible to anything else. Never by itself, but always through the mediation of another. How can one claim that worlds are untranslatable, when translation is the very soul of the process of relating? (Latour, 1993, p. 113, our emphasis).

The task acceding to Latour is therefore to identify the instruments of translation at work in particular acts of commensuration.

In the next section we will examine the key instruments of translation in KT. To understand how they operate, however, we must first relate these instruments of translation to its underpinning epistemology, evidence-based medicine (EBM).

\section{The translation instruments of $\mathbf{K T}$}

$\mathrm{KT}$ is often seen as an operationalization of the epistemological premises of EBM, making research evidence available for clinical use through translation. Or, as one paper has it in the title, KT is about 'shortening the journey from evidence to effect' (Davis et al., 2003, our emphasis). KT is, then, the instrument used in this 'shortening'.

Around 2000, several researchers drew attention to the fact that many innovative research results never left the laboratory setting. There was an increased attention to the 'disconnection between the promise of basic science and the delivery of better health'. It was this recognition that inspired a completely new range of knowledge production in medicine, which now increasingly has been referred to as 'translation' (Sung et al., 2003). This concern with evidence and its social and clinical efficiency was a product of a new biomedical paradigm that gradually became hegemonic in the post-war period. The Canadian Institute of Health Research was a pioneer in conceptualizing KT, and it is hardly a coincidence that KT was first defined in Canada. It was here also that the shift towards evidencebased medicine (hereinafter EBM) first began (Solomon, 2015).

The epistemological premise of EBM is that all knowledge is inherently biased, and that such bias needs to be balanced by intersubjectively valid expert claims. Characteristic of the epistemological paradigm of EBM is the idea that knowledge is independent of the expert. Hence, knowledge does not dwell in the body of the 'initiated' doctor, but in a textual world available to the expert and the layperson alike (Timmermans and Berg, 2010). Accordingly, everyone may become an 'expert' by drawing on the 'best evidence'. This, moreover, is made democratically available through systematic reviews. Hence, the EBM paradigm actively seeks to undermine the personal and/or 'traditional' authority of medical doctors and experts, and to control bias and prejudice by constructing a new textual authority, accessible to people outside the medical profession, which in turn rests on basic science. Until the 1950s, the medical doctor was the embodiment of knowledge, and s/he did not need any documentation of his or her epistemological authority, except the degree and the professional experience (Weisz et al., 2007).

Randomized controlled trials (hereinafter RCTs) are often referred to as the 'gold standard' of modern clinical medicine. (A well-known example is the comparison of the effects on groups of patients that are offered a specific intervention or drug with a group that is given a placebo.) With the introduction of RCTs in the 1950s, knowledge became increasingly independent of the observer. It was, we could say, dislocated from the individual professional, and relocated in institutions and textual forms, like expert panels and clinical guidelines. A further consequence of this was that medical evidence within this development was considered translatable through texts and accessible to everyone (we will return to this in more detail below). 
Three entangled processes in the history and sociology of medical knowledge were central in the development of translation as a medical research area. These processes also developed three instruments of medical translation, which constructed new 'yardsticks' or 'measuring measures' for the production of medical knowledge, as well as its social application (cf. Latour above). These instruments of translation were:

RCTs. Firstly, a set of new research methods were developed. In sum, these research methods formed a supplement to the two traditional fields of medical research, namely basic research and clinical research. It is crucial that the translational methods in question allowed for a stepwise testing of findings and results developed in the laboratory setting, first on animals, and then on humans, (originally, to test the efficacy of drugs). The aim of this new translational research was to integrate methods and knowledge from basic sciences, such as genetics and molecular biology, into clinical interventions, which could be further tested through RCTS. To gain clinical significance, it was claimed, results from laboratory research should be prepared for testing on large populations in RCTs (Solomon, 2015).

Literature reviews. Secondly, new text genres grew from the development of RCTs. Proponents of KT and translational research devised a set of textual genres with the aim of summarizing research for clinical use. Different forms of cognition and text production were set to work; both content analysis (in systematic literature reviews) and statistical analysis (meta-analysis) were introduced as a manner of evaluating the RCTs of a particular treatment or intervention. Moreover, this textualizing of medical knowledge formed the basis for so-called clinical guideline recommendations. In these texts, reviews of several trials were operationalized into normative practical guidance for clinicians. Through the new guideline methodology, based on statistical meta-analysis of clinical trials, evidence became detached from the expert. (Timmermans and Berg, 2010; Solomon, 2015).

Clearing Houses. Thirdly, new institutions were established, bringing together scientists, clinicians and bureaucrats in socalled 'Clearing Houses' or 'knowledge centres', with the aim of facilitating the production of research reviews. Guideline development groups were also established, either as new institutions or as departments in already established units, such as the national health agencies, to promote and organize the development of guidelines (Weisz et al., 2007). An example of one such institution is the Cochran Collaboration in Oxford, certainly the most famous Clearing House, which organized medical research information in systematic ways. Another important epistemic practice was holding consensus meetings, taking place primarily in the 1980s and 1990s (Solomon, 2015). Here, specialists came together to discuss contested issues and make clinical recommendations. These meetings were, in one way, a result of this new realization of knowledge bias (thought to be compensated for by the presence of many experts), but on the other hand, they were still based on the authority of expert opinions.

With the rise of evidence-based medicine and the concomitant idea of an evidence-based practice, medical knowledge became translatable in a new way. The ability to translate-from basic science to practical healthcare action-is now the key to the implementation of medical knowledge. Although very different, all these methodological, technical, and textual practices share the common purpose of translating knowledge from 'bench to bedside', or from basic research into clinical practice.

\section{The cultural model of KT}

We have observed that KT has been conceptualized as a process with distinct stages: the production of knowledge (basic research); the testing of knowledge (RCTs); and the dissemination of scientifically warranted knowledge (guidelines). The most common current model of how these separate stages should be integrated in the process of translation comprises three stages, moving from the production of scientific knowledge to its application:

T1: A passage from basic science in laboratories to clinical research on populations (this is also known as translational research, cf. above), and further on

T2: A passage from clinical research to clinical recommendation, often in terms of the development of clinical guidelines based on systematic reviews of clinical trials, and ending in

T3: A passage from clinical recommendations to routine clinical practice (Woolf, 2008).

In this model, medical translation of knowledge is construed as a process of testing and synthetizing scientific results produced in the laboratory, to prepare it for sound clinical application and scientifically warranted healthcare. The process begins in a place emblematic for modern science, the laboratory, with basic science ('pure science' in its most heroic form, we could say). The directionality of translation is from the place where science is produced to its application. Thus, translation is viewed as a linear process of knowledge production and transmission. Another influential model is the Knowledge-to-Action-Model developed by the Canadian Institute of Health Research. This proposes a knowledge creation pyramid divided into three stages of knowledge creation (knowledge inquiry, knowledge synthesis and knowledge tools), which is set to interact with what the institute calls an 'application cycle' (see figures in Graham et al., 2006). Here, the application of knowledge is depicted as spatially external to the production of knowledge.

In both the cited models, the aim is that concrete practices should be governed by science, and that application should follow from, and be a supplement to, knowledge creation. This is illustrative of the epistemology underpinning KT. In KT, translation is-if it is to be felicitous-non-productive; it should neither add to nor detract from the evidence and findings produced by basis research and RCTs. On the contrary, the purpose of translation in $\mathrm{KT}$ is to preserve and implement the original, scientific content in new socio-cultural contexts, resulting in (assumed) rational governance and practical healthcare in particular cases around the globe.

Using the idiom of translation studies to describe $\mathrm{KT}$, we can say that the act of translation is a process of 'copying the original' where the translational act itself is a non-act, the translator a nonactor, and the purpose of translation is to be a 'container' of the original message, without adding, transforming or in any other way 'betraying' the original. Moreover, this lack of attention to textual and cultural factors is due to an uncritical acceptance of cultural models of translation, knowledge production and knowledge dissemination, that combine elements from two of the most persistent paradigms in the European history of ideas, namely Romanticism and Enlightenment ideas. In the case of KT, we find a combination of

- (a) notions from esthetic and literary Romanticism: translation is the art of preserving, while 'carrying across', the artistic genius behind the original masterpiece

- (b) an unquestioned Enlightenment model of knowledge dissemination: knowledge should trickle down from elites and theory into 'popular' practice, the bedside of everyday care. 
Thus, it is possible to identify a set of persistent cultural models of knowledge, its creation, communication, and transmission at work in KT. Referencing G. Steiner, we could say that this manner of patterning knowledge and translation constitutes a topological constant, which 'remain invariant when that figure [in our case, 'translation'] is bent out of shape' (Steiner, 1975, pp. 448-449). KT, then, distributes value and translational directionality in ways that resemble ancient literary and philosophical ideologies of translation: The original is the source of value, and its admired qualities should be kept intact in every process of translation.

The epistemology behind such a view of knowledge and translation assumes that it is possible to separate the production of knowledge from its transfer. Accordingly, the scientific content to be translated is construed as being outside the process of translation; it belongs to a separate 'cycle of action' and 'application' (cf. citation of Graham et al., 2006 above). Knowledge, moreover, is said to have reached its culmination in the secluded space of the laboratory or the more mobile 'seclusions' of RCTs (testing the effect, transferability, reproducibility and relevance of knowledge), and it is the findings that should be transported to, and implemented in, situations of practical care. In line with this, so-called barriers and drivers of KT are essentially understood as social and cultural factors external to the production of knowledge (Davis et al., 2003). 'Drivers', we could say, helps knowledge keep its identity, and thus remain equivalent to the original 'ST' of the laboratory, while 'barriers' threaten to insert shifts and difference into the 'original message' produced in the laboratory. In the next section, we claim that an attention to the inevitable supplement of translation offers a model for tackling this constitutive dilemma of KT.

\section{Supplementing KT with new models for translation}

We have observed how various textual genres are mobilized in the different stages of the KT process, beginning in literary reviews and ending in so-called clinical guidelines, which prescribe manners of intervention in concrete cases (e.g., particular diagnoses), based upon the systematic reviews of the scientific state of the art as this is expressed in the literature. Hence, the translation process hinges upon textualization in such genres as systematic reviews and guidelines. These texts, moreover, in increasingly condensed and vernacular forms, must be able to transmit the science necessary to implement state-of-the-art care. Generally, this text production is conceived as supplementing a lack of knowledge among clinical practitioners. Thus, what we will call a textual supplement, namely a concern about target audiences (practitioners and patients), is inevitable, even in the creation of an 'autonomous' science restricted to the body as a bio-medical phenomenon.

It is our contention that $\mathrm{KT}$, in its current forms, is based on an inadequate understanding of the textual and cultural supplements that affect the construction, dissemination, and application of knowledge (Engebretsen et al., 2020, cf. Derrida, 1997 on 'supplement'). In translation studies, the importance of cultural factors in translation has been thoroughly emphasized. A case in point is André Lefevere, who maintained that problems in translation are not primarily of a linguistic nature. Rather, questions of translatability have more to do with 'discrepancies in the conceptual and textual grids', than with 'discrepancies in languages' (Lefevere, 1999). Take the phrase 'once upon a time', the interpretation of which requires knowledge of a particular cultural genre, the fairytale. Cultural and textual framings such as this marker of genre cannot be read out of the sentence as 'mere linguistic data'; that is, the phrase 'once upon a time' does not per se signify 'this is fairytale' and 'this is fiction'; to understand that the phrase serves as a generic code signaling a suspension of ordinary reality requires cultural competence. Even linguistic translation, then, must also account for cultural factors, and 'metadiscursive practices' for producing, classifying and interpreting messages in various genres (cf. Briggs, 1993).

In philosophy, Jacques Derrida has radically asserted that translation is an integral part of all textual production. The translation, or target text, relates to the source text, in what Derrida has referred to as the logic of the supplement: it both adds on to the original and compensates for a lack in the original (Derrida, 1997). According to Derrida, the nature of the supplement is ambiguous, by both adding onto itself, being 'a plenitude enriching another plenitude, the fullest measure of presence', and completing something that is missing 'the supplement supplements... adds only to replace' (Derrida, 1997, p. 44). Consequently, a translation does not only duplicate the original message, but it also completes the original message, by fulfilling one of the ST's possible interpretations. This, moreover, implies that the necessity of interpreting and translating textsthe fact that texts do not speak for themselves, but are constantly objects of interpretation, and are scrutinized for their true meaning-'always already' characterizes the ST.

If supplementary interpretations and semantic shifts are an inevitable outcome of the transport of signs between texts, KT could become more effective if such shifts were defined as a creative potential rather than as a mere 'barrier'. Accordingly, it is our contention that KT relates to the 'original scientific content' as a double supplement.

- On the one hand, KT offers new approaches to the communication of scientific knowledge to different groups in the healthcare system, with the aim of supplementing a lack of knowledge among clinicians (and patients).

- On the other hand, it demonstrates that a textual and cultural supplement, namely a concern with target audiences (clinicians and patients), is inevitable even in the creation of an 'autonomous' science. The individual patient is both the origin and the end of medicine.

Translation therefor both threatens and fulfills the original scientific message. This dual challenge from translation can be used to identify an inherent paradox in existing KT models. While these models presuppose that the principal duty of adequate $\mathrm{KT}$ is to implement the original scientific message in new social contexts and textual forms, without altering its content, the same models, paradoxically, also state that it is through translational modifications and adaption to new audiences, through synthesis and development of guideline recommendations, that the message becomes scientifically trustworthy and effective. It is in this sense that translation is simultaneously a threat, and the instrument, that is supposed to realize the potential of the original scientific message. However, existing KT models fail to draw the consequences from this paradox: translation is inherent in science and the division between science and its translation is both impossible and unproductive to maintain (cf. Engebretsen et al. 2017).

\section{Clinical inference-singularity in culture and medicine}

K. Popper maintained that science was all about problem solving. If $\mathrm{KT}$ is seen as 'problem solving', the problem it aims to solvehow to relate 'hard', natural, and biomedical science to practical healthcare-also implies a crossover between disciplinary domains that modernization theory claims have been separated in modernity. A case in point is Latour, who claims that modern knowledge production is based upon a construction of nature and 
culture as separate, ontological domains with concomitant epistemological approaches (focused upon causes and meaning respectively). Culture is a product of human creativity, while nature exists in a realm beyond the sphere of human productivity (natural laws work independently of human agency). Characteristic of the 'modern', however, are also the continuous processes of translation and meditation that link nature with culture/ society. But these translations are balanced by processes of 'purification' that reestablish the borders between natural and cultural knowledge. Together these interacting processes create 'hybrids' of nature and culture that makes technological modernity work (Latour, 1993, pp. 10-12).

Clearly, Latour's description fits KT well: The KT translation process is a continuous attempt at keeping natural, biomedical science 'pure', while simultaneously making it into a 'hybrid' by turning medical knowledge into practical care. However, our analysis of KT also suggest a twist in relation to the binaries of modernization theory; it demonstrates that an orientation towards a target audience, clinicians, and patients, is inevitable, even in the creation of an 'autonomous' science. This is so because the individual patient is both the origin and the end of medicine, at least in the sense that curing, and caring for, individual human beings is the shared objective of medical practice and research. On the one hand, this surely shows that $\mathrm{KT}$ and medicine are 'hybrid' forms of knowledge through and through. On the other, it also demonstrates that medicine, as a practice, has an inherent relation to singularity that the language of modernity theory fails disregards (cf. Kristeva et al., 2018). We should not map the opposition between the nomothetic and the singular onto the opposition between the natural and the human sciences. The use the anthropologist Clifford Geertz made of practical medical reasoning to 'free' anthropology from a nomothetic social science can serve as a case in point.

In the introduction to the The Interpretation of Culture, Geertz used the medical notion of clinical inference to construct a new model for reading culture (Geertz, 1973). Clinical inference comes into play in situations where a medical practitioner meets individual patients manifesting specific symptoms. To make a diagnosis, the practitioner needs to relate these symptoms to a more general system of medical knowledge. Geertz' application of a medical analogy has mostly escaped commentators, who have turned their attention towards the seminal text analogy, which enabled Geertz to study cultures as if they were texts. The text analogy enabled Geertz to align anthropology with a 'soft' humanistic approach-and marked his distance to the 'harder' social sciences, modeled upon the natural sciences. Geertz wanted to develop a meaning-centered and interpretative approach to the study of culture. To do this he turned to hermeneutics, often regarded as a mark of the 'soft' human sciences dealing with interpretation, in contrast to the 'hard', natural sciences-dealing with facts.

In the context of $\mathrm{KT}$, it is therefore highly relevant that Geertz developed the text analogy as the basis for an interpretative anthropology that aimed at reading culture by turning to clinical inference, a form of practical reasoning from medicine. As we have seen, KT was born as a consorted scientific and governmental attempt to take control of the clinical inference and singularity of case-based reasoning that Geertz used to establish an interdisciplinary common trait between (clinical) medicine and interpretative anthropology. Based on clinical inference as a particular form of medical and diagnostic form of observation, description and interpretation, Geertz establishes a pattern of inference shared across the divide between the natural and the human sciences:
To generalize within cases is usually called, at least in medicine and depth psychology, clinical inference. Rather than beginning with a set of observations and attempting to subsume them under a governing law, such inference begins with a set of (presumptive) signifiers and attempts to place them within an intelligible frame. Measures are matched to theoretical predictions, but symptoms (even when they are measured) are scanned for theoretical peculiarities-that is, they are diagnosed. In the study of culture, the signifiers are not symptoms or clusters of symptoms, but symbolic acts or clusters of symbolic acts, and the aim is not therapy but the analysis of social discourse. But the way in which theory is used-to ferret out the unapparent import of things-is the same (Geertz, 1973, p. 26, our emphasis).

Here, the cognitive common trait between medicine and a hermeneutic study of culture is the search for implicit meanings of symptoms and signifiers within a local context (a body, an etiology, a culture) by teasing out 'the unapparent import of things'. It is such 'unapparent import' that may be lost if researchers hasten to subsume observations under general laws applicable across cases, rather than dwell on seemingly unimportant symptoms and signs.

Why should we bother with such assumed similarities to expand current notions of KT? This reading of Geertz tells us that medicine as a science-as a knowledge practice aiming for the practical effect of healing, caring, and curing-does not fit neatly into a clearly cut division of disciplines into the nomothetic and ideographic, general laws and singular events. This is so because the beginning and end of medicine as a total sociocultural and scientific fact is the singular existence of the individual patient, as what we could call a 'biocultural unit' (cf. Kristeva et al., 2018). Hence, we should not map the opposition between the nomothetic and the singular onto the opposition between the natural and the human sciences; medicine oscillates between nature and culture as well as the singular and the general.

\section{Conclusion}

In this introduction, we have claimed that the practice of KT might benefit from incorporating more theoretical notions of translation as an entangled material, textual and cultural process which inevitably impacts the 'original scientific message'. It is our contention that concepts and practices of translation have an unexploited potential for bridging the gap between medicine and social/human sciences. Such interdisciplinary exchange can, in turn, contribute to an increased understanding of the interplay between medical cultures and the target cultures of KT, and thereby ultimately enhance the flow of knowledge within healthcare. Hence, rather than dropping the KT metaphor, we should extend it by taking advantage of discourses and practices of translation in the human sciences.

Received: 15 February 2022; Accepted: 15 February 2022; Published online: 04 March 2022

\section{Notes}

1 This introduction is based upon but expands these earlier works on KT and translation.

2 'Translatio' and 'transferre' are Latin translations of the Greek 'metaphora' and 'metaphero'; the name of the trope refers to the act of carrying something across a boundary. Thus, (still etymologically speaking) 'translatio' simply implies that some agent carrying some (undefined) thing crosses a spatial boundary, and the transferred object does not have to be linguistic (Cheyfitz 1997, p. 35; Evans, 1998). 


\section{References}

Bergman A (1984) L'épreuve de l'étranger: Culture et traduction dans l'Allemagne romantique. Gallimard, Paris

Briggs C (1993) Metadiscursive practices and Scholarly Authority in Folkloristics. J Am Folkl 106(422):387-434

Buden B, Nowotny S (2009) Cultural translation: an introduction to the problem. Transl Stud 2(2):196-298

Burke P (2007) Cultures of translation in early modern Europe. In: Burke P, PoChia Hsia R (eds) Cultural translation in early modern Europe. Cambridge University Press, Cambridge, pp. 7-38

Cheyfitz E (1997) The poetics of imperialism: translation and colonization from The Tempest to Tarzan. University of Pennsylvania Press, Philadelphia

Davis D, Davis ME, Jadad A et al. (2003) The case for knowledge translation: shortening the journey from evidence to effect. PubMed Central 327(7405):33-35

Derrida J (1997) Of grammatology. Johns Hopkins University Press, Baltimore

Engebretsen E, Ødemark J, Sandset T (2017) Expanding the knowledge translation metaphor. Health Res Policy Syst 15(1). https://doi.org/10.1186/s12961-0170184-x

Engebretsen E, Henrichsen G, Ødemark J (2020) Towards a translational medical humanities: introducing the cultural crossings of care. Med Humanit 46(2). https://doi.org/10.1136/medhum-2019-011751

Evans R (1998) Metaphor of translation. In: Baker M (ed) Routledge encyclopedia of translation studies. Routledge, London, pp. 149-152

Geertz C (1973) The interpretation of cultures. Basic Books, New York

Graham ID, Logan J, Harrison MB, Straus SE, Tetroe J, Caswell W, Robinson N (2006) Lost in knowledge translation: time for a map? J Contin Educ Health Prof 26(1):13-24

Greenhalgh T, Wieringa S (2011) Is it time to drop the 'knowledge translation' metaphor? A critical literature review. J R Soc Med 104:501-509

Kristeva J, Moro MR, Ødemark J, et al. (2018) Cultural crossings of care: An appeal to the medical humanities Medical Humanities 44:55-58. https://doi.org/ 10.1136/medhum-2017-011263

Latour B (1993) We have never been modern. Harvard University Press, Boston

Lefevere A (1999) Composing the other. In: Bassnett S, Trivedi H (ed) Postcolonial translation: theory and practice. Routledge, London, pp. 75-94

Machen R (2018) Towards a critical politics of translation: (Re)producing hegemonic climate governance. Nat Space 1(4):494-515

Mankoff SP, Brander C, Ferrone S, Marincola FM (2004) Lost in translation: obstacles to translational medicine. J Transl Med 2(1):14

Nida EA, Taber CR (1969) The theory and practice of translation, with special reference to Bible translating. Brill, Leiden

Ødemark J, Henrichsen G, Engebretsen E (2021) Knowledge translation. In: Sebnem S, Spišiaková E (eds) The Routledge handbook of translation and health. Routledge, London, pp. 149-161

Severi C, Hanks WF (2015) Introduction. In: Severi C, Hanks WF (eds) Translating worlds: the epistemological space of translation. Hau books, Chicago, pp. 1-17

Solomon M (2015) Making medical knowledge. Oxford University Press, Oxford

Steiner G (1975) After Babel: aspects of language and translation. Oxford University Press, Oxford

Straus S, Tetroe J, Graham I (2009) Defining knowledge. Can Med Assoc J 181(34):165-168

Sung NS, Crowley Jr WF, Genel M et al. (2003) Central challenges facing the national clinical research enterprise. JAMA 289:1278-1287

Timmermans S, Berg M (2010) The gold standard: the challenge of evidence-based medicine and standardization in health care. Temple University Press, Philadelphia
Venuti L (1995) The translator's invisibility. Routledge, London

Venuti L (2009) From translation, community, utopia. In: Damrosch D, Melas N, Mbongiseni B (eds) The Princeton sourcebook in comparative literature. Princeton University Press, Princeton, pp. 358-379

Weisz G, Cambrosio A, Keating P, Knaapen L, Schlich T, Tournay VJ (2007) The emergence of clinical practice guidelines. Milbank Q 85(4):691-727

Wintroub M (2015) Translations: words, things, going native and staying true. Am Hist Rev 120(4):1185-1217

Woolf SH (2008) The meaning of translational research and why it matters. JAMA 299(2):211-213

World Health Organization (2019) Ageing and life course. Knowledge translation. https://www.who.int/ageing/projects/knowledge_translation/en/. Accessed 7 June 2019

\section{Acknowledgements}

The authors acknowledge the support and funding from the Centre of Advanced Studies at the Norwegian Academy of Sciences and Letters and the Research Council of Norway, project number 315928 .

\section{Competing interests}

The authors declare no competing interests.

\section{Ethical approval}

Not applicable.

\section{Informed consent}

Not applicable.

\section{Additional information}

Correspondence and requests for materials should be addressed to John Ødemark

Reprints and permission information is available at http://www.nature.com/reprints

Publisher's note Springer Nature remains neutral with regard to jurisdictional claims in published maps and institutional affiliations.

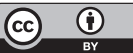

Open Access This article is licensed under a Creative Commons Attribution 4.0 International License, which permits use, sharing, adaptation, distribution and reproduction in any medium or format, as long as you give appropriate credit to the original author(s) and the source, provide a link to the Creative Commons license, and indicate if changes were made. The images or other third party material in this article are included in the article's Creative Commons license, unless indicated otherwise in a credit line to the material. If material is not included in the article's Creative Commons license and your intended use is not permitted by statutory regulation or exceeds the permitted use, you will need to obtain permission directly from the copyright holder. To view a copy of this license, visit http://creativecommons.org/ licenses/by/4.0/

(c) The Author(s) 2022 